BULLETIN (New Series) OF THE

AMERICAN MATHEMATICAL SOCIETY

Volume 38, Number 4, Pages 389-408

S 0273-0979(01)00913-2

Article electronically published on July 12, 2001

\title{
A MAD DAY'S WORK: FROM GROTHENDIECK TO CONNES AND KONTSEVICH THE EVOLUTION OF CONCEPTS OF SPACE AND SYMMETRY
}

\author{
PIERRE CARTIER
}

\section{INTRODUCTION}

To add to the chorus of praise by referring to my own experience would be of little interest, but I am in no way forgetting the facilities for work provided by the Institut des Hautes Études Scientifiques (IHES) for so many years, particularly the constantly renewed opportunities for meetings and exchanges. While there have been some difficult times, there is no point in dwelling on them.

One of the great virtues of the institute was that it erected no barriers between mathematics and theoretical physics. There has always been a great deal of interpenetration of these two areas of interest, which has only increased over time. From the very beginning Louis Michel was one of the bridges due to his devotion to group theory. At present, when the scientific outlook has changed so greatly over the past forty years, the fusion seems natural and no one wonders whether Connes or Kontsevich are physicists or mathematicians. I moved between the two fields for a long time when to do so was to run counter to the current trends, and I welcome the present synthesis.

Alexander Grothendieck dominated the first ten years of the institute, and I hope no one will forget that. I knew him well during the 50s and $60 \mathrm{~s}$, especially through Bourbaki, but we were never together at the institute - he left it in September 1970 and I arrived in July 1971. Grothendieck did not derive his inspiration from physics and its mathematical problems. Not that his mind was incapable of grasping this area - he had thought about it secretly before 1967-but the moral principles that he adhered to relegate physics to the outer darkness, especially after Hiroshima. It is surprising that some of Grothendieck's most fertile ideas regarding the nature of space and symmetries have become naturally wed to the new directions in modern physics. It is this unexpected marriage - and its occasionally comical aspects - that I would like to talk about here. "A mad day's work", as you know, is the subtitle given to The Marriage of Figaro by Beaumarchais. From a certain distance there is less cause for astonishment; the concepts of space and symmetry are so fundamental

Received by the editors August 15, 2000.

2000 Mathematics Subject Classification. Primary 00B25.

Key words and phrases. Institut des Hautes Études Scientifiques, point, bundle, category, Boolean algebra, motive.

Translated from the French by Roger Cooke. This paper was written on the occasion of the 40th anniversary of the IHES (in 1998) and was included in the corresponding Festschrift volume Les relations entre les mathématiques et la physique théorique (distributed to the participants, but never published). We thank the IHES for permission to reprint it. 
that they are necessarily central to any serious scientific reflection. Mathematicians as influential as Bernhard Riemann or Hermann Weyl, to name only a few, have undertaken to analyze these concepts on the dual levels of mathematics and physics.

\section{A BRIEF BIOGRAPHY of GrothendiecK}

Grothendieck wrote a long, very personal memoir, Harvesting and Sowing (which remains to date unpublished), but it would be difficult to find a narrative of his life in it, especially his childhood. In the three volumes of the Grothendieck Festschrift that I published on the occasion of his sixtieth birthday there is a brief introduction and a rather long analysis by Dieudonné of Grothendieck's work. The narrative of his life there is rather cursory, besides being abridged in relation to his early projects. In his autobiography [10] Laurent Schwartz mentions his student Grothendieck, but only in passing and with many inaccuracies. What I know of his life comes from Grothendieck himself, and that, supplemented by some other testimony, forms the basis of the following narrative.

First of all, I should mention the remarkable personality of his parents. His father's name was Shapiro-I don't know his first name. He was born around 1890 in a small town close to the point where Russia, Ukraine, and Belarus now meet. All the Shapiros (there are numerous spellings of the name: Shapira, Szpiro, ... ) are descended from a group in a very small geographical region. Alexander Grothendieck's grandfather was probably a member of the community of Hasidic Jews to which the Shapiros belonged. These were very pious Jews, who would nowadays be called fundamentalists. Some of them were so "enamored of God" that they had themselves walled into a small tower with a window where the faithful came to offer them alms of food. While visiting Grothendieck I have seen a portrait of his father, done by a co-detainee in the French camp of Vernet in 1942. It bears a strong resemblance to the photograph of the son that we put at the front of the Festschrift, the head shaved, with a fiery expression of the eyes.

As Alexander told me, his father's political career constitutes a Who's Who of the European revolution from 1900 to 1940. Because of the borders that existed at the time, he was born a Russian citizen. He participated in the abortive 1905 revolution against the tsar, along with the revolutionary currents of the period. After the quashing of the revolution he was deported to Siberia, and spent more than ten years in jail. He was released in 1917, when the exiles returned to Russia to work for the overthrow of the Russian monarchy. Two revolutions occurred in St. Petersburg: the Menshevik Revolution of February 1917 and the Bolshevik Revolution of October 1917. Shapiro was one of the leaders of the "SocialistRevolutionary of the Left" Party. Allied at first with the Bolsheviks in October 1917, they soon clashed with Lenin and formed part of the many revolutionariesBolsheviks and non-Bolsheviks alike - purged by Lenin 1

After the collapse of the two central empires, Europe was agitated by a number of revolutionary movements: Rosa Luxemburg and the Spartakists in Berlin in 1919, the Munich Soviets, Bela Kun's revolution in Hungary. To these we may add the various events of the Russian Civil War, one of them being the Makhno movement ${ }^{2}$ in Ukraine. Grothendieck's father participated in all these movements.

\footnotetext{
${ }^{1}$ Shapiro is portrayed in the famous book of John Reed, Ten Days That Shook the World, a slightly romanticized account of the revolution of October 1917.

${ }^{2}$ From 1920 to 1924 Ukraine enjoyed a brief independence. The war there was a tripartite war among the Red Bolsheviks, the White Tsarists, and the Makhnovists. This last movement
} 
During the 1920s he lived mostly in Germany, participating in the political and armed clashes of the leftist parties against Hitler and his Nazis. In Germany he met Hanka Grothendieck, a Jewish woman from the north of Germany 3 In Berlin on March 28, 1928, she had a son by Shapiro, the hero of our story. I know very little about her previous child, an elder sister to Alexander. But very soon Hitler came to power. After 1933 Germany was too dangerous for revolutionary Jews, and the couple fled to France, leaving their son hidden in a libertarian private boarding school near Hamburg.

In 1936 the Spanish Civil War broke out. Shapiro, like Simone Weil (sister of the mathematician André Weil), joined the anarchist militias of the F.A.I. in the struggle against Franco's Fascists. Hanka Grothendieck seems to have remained in France during this time. Whatever the case, she sent for her son in 1938. This was the time of the rout of the Spanish Republicans, who fled to the Midi. Refugee camps were opened, some of which became detention camps-I would not go so far as to say concentration camps. At Vernet and at Gurs in the Pyrenées camps were hastily formed to hold temporarily all the "dangerous foreigners": German Jews, Spanish anarchists, and Trotskyites 4 The declaration of war on September 3, 1939, did nothing to improve the lot of these outcasts, as one can well imagine. After the capitulation in June 1940 many prisons were opened, among them that of André Weil in Rouen, and the Grothendieck family was temporarily out of confinement. Starting in October 1940 the Vichy government of Marshal Pétain promulgated anti-Jewish laws that were also enforced in the unoccupied zone. Shapiro was brought to the Vernet camp, and with other detainees, sent directly to Ausschwitz, where he died in 1942 - one of the most shameful episodes in French history.

Grothendieck and his mother survived only with difficulty in anti-Semitic Vichy France. Their salvation came from the Protestant resistance movement in Cévennes. Pastor Trocmé, director of the private Protestant Lyceum in Chambon-sur-Lignon (called Collège Cévenol), transformed this charming vacation resort, frequented by high Protestant society, into a center of both spiritual and military resistance to the Nazi occupiers 5 Grothendieck was a pupil at Collège Cévenol and was housed at the Swiss Foyer, which gave shelter to proscribed children of all origins 6 After receiving the "baccalauréat" degref 7 and, I think, with Protestant recommendations, he became a student at Montpellier. It has often been said that he was ahead of his teachers and that he was already exhibiting a taste for extreme generalization in mathematics. He arrived in Paris, having finished his licensure, in the

combined some aspects of peasant jacquerie with Ukrainian nationalism. The Spanish anarchists claimed it, rightly or wrongly, as a model of a spontaneous peasant uprising. In the nowindependent Ukraine, Makhno is revered as a national hero, at least by the more nationalist parties.

${ }^{3}$ I have often been asked if the name "Grothendieck" is Dutch. The "plattdeutsch" of northern Germany is linguistically close to Flemish.

${ }^{4}$ Contrary to frequent claims in French propaganda, these camps functioned from late 1938 on, not starting in September 1939. On the latter date new camps were opened for "enemy nationals".

${ }^{5}$ The Wehrmacht never dared to risk going into these mountainous regions, where people prided themselves on having learned the spirit of resistance after the revocation of the Edict of Nantes in 1684 !

${ }^{6}$ In the 1960s I made the acquaintance of a former teacher at Collège Cévenol who was on the eve of retirement. I arranged a meeting between Grothendieck and his former teacher, which was a very emotional experience for both of them.

${ }^{7}$ In French parlance, that is the final exam at high school, giving access to all French universities. 
autumn of 1948, equipped with a letter of recommendation to Élie Cartan 8 from his Montpellier teacher.

Here ends the infancy narrative of the gospel and begins the public career of the prophet. It was to last from 1949 to 1970 . It is too well known for me to repeat it, and we may refer to the narrative of Dieudonné, even if it is somewhat brief. We shall mention only that Grothendieck was interested in functional analysis from 1950 to 1957, that his dissertation [7] is a masterpiece, but that the article that had the most subsequent influence was undoubtedly [8, the point of departure of the geometric theory of Banach spaces. Then, from 1956 to 1970, he completely reworked homological algebra and algebraic geometry. His scholarly career essentially ends at that point.

I would like to try to analyze the reasons for this abrupt end to a career so astonishing and fertile at the age of 42 . The reason given was that he had discovered that the Ministry of Defense had been subsidizing the institute. The IHES's finances are less obscure nowadays than they were in the time of Motchane, and, as far as I know, there is still today a modest financial line marked D.R.E.T 9 In order to understand the vehemence of Grothendieck's reaction, one must take account of his past and the political situation of the time. He is the son of a militant anarchist who had devoted his life to revolution. This was a father of whom he had very little direct knowledge; he knew him mostly through his mother's adulation. He lived as an outcast throughout his entire childhood and was a "displaced person" 10 for many years, traveling with a United Nations passport (also known as a Nanssen passport). He had always been uncomfortable frequenting the "better" places and felt more at ease among the poor, even the impoverished. The solidarity of outcasts had created in him a strong feeling of compassion. He lived his principles, and his home was always wide open to "stray cats". In the end he came to consider Bures a gilded cage that kept him away from real life. To this reason he added a failure of nerve, a doubt as to the value of scientific activity. Starting in 1957 at a Bourbaki Congress, he confided his doubts to me and told me that he was considering activities other than mathematics 11 One should perhaps add the effect of a well-known "Nobel syndrome". After his dedication at the Moscow Congress in 1966, where he received the Fields Medal, when he was laboring over the last (decisive) stages of the proof of the Weil conjectures and perhaps beginning to perceive that Deligne would be needed to complete in 1974 the program he had set for himself, and perhaps yielding to the pernicious view that sets 40 as the age when mathematical creativity ceases, he may have believed that he had passed his peak and that thenceforth he would be able only to repeat himself with less effectiveness.

The mood of the time also had a strong influence. The disaster that had been the second Viet Nam War from 1963 to 1972 had awakened many consciences. All around him his friends were struggling against the Viet Nam War in great numbers;

\footnotetext{
${ }^{8}$ The famous geometer Élie Cartan was already aged and feeble, but his son Henri Cartan was the rising star (and the godfather) of French mathematics in the 1940s. Henri Cartan always maintained close relations with the Protestant areas in the south of France and was the natural host for Grothendieck in Paris.

${ }^{9}$ Acronym for "Direction de la Recherche et des Études Techniques", a small funding agency of the Ministry of Defense.

${ }^{10}$ His citizenship papers disappeared in the Berlin apocalypse of 1945.

${ }^{11}$ His mother wrote poems and novels, in German, of course. He was thinking of pursuing this route.
} 
a significant number of French mathematicians took concrete action and traveled to Hanoi, as he (and I) did. The student uprising of 1968 (in France) was to a large extent the result of this movement. Its anarchist aspect held attractions for him and forced him to admit that he had ceased being an outcast and had become a scientific mandarin. The movement of May 1968 aroused others among the Bourbaki group, such as Chevalley, Samuel, and Godement. The cold war was at its height, and the risk of a nuclear confrontation was very real. The problems of overpopulation, pollution, and uncontrolled development - everything that is now classified as ecology - had also begun to attract attention. There were plenty of reasons to call science into question!

He reacted with his impetuous nature and founded the small "Survival" sect. On more than one occasion, he exasperated those who shared his politics with his excesses and his inept tactics 12 His route is quite close to that of Simone Weil, and political anarchism took on more and more a religious tone in him. But, while Simone Weil's Catholicism was violently anti-Semitic (in 1942!), Grothendieck's Buddhism bears a strong resemblance to the practices of his Hasidic ancestors 13 For a long time he was receptive to all sorts of marginal "hippies", which resulted in his indictment and an absurd trial in 1977 due to a 1945 regulation that made it a misdemeanor to meet with a foreigner. He enjoyed playing the role of a modern Socrates, and was given a suspended sentence of six months in prison and a fine of 20,000 francs. It seems to me that his definitive break with science dates from this incident. He withdrew more and more into his own tent. After 1993 he no longer had a postal address and settled in a hamlet in the Pyrenées. Very few managed to see him there. If I can believe his most recent visitors, he is obsessed with the Devil, whom he sees at work everywhere in the world, destroying the divine harmony and replacing $300,000 \mathrm{~km} / \mathrm{sec}$ by $299,887 \mathrm{~km} / \mathrm{sec}$ as the speed of light!

He remains, however, the same Grothendieck who contributed to the renown of the IHES during its first ten years of existence. He is the same person to whom we owe the magnificent ideas on space and symmetry that I am now about to develop.

\section{ON THE NATURE OF SPACE AND ITS POINTS}

The central problem is that of the points of space. The debate goes back to Leibniz and Newton. For Leibniz the constituent of all things, both material and spiritual, is provided by monads, which are windowless (we would say they have no internal structure), and the only things that matter are their mutual relationships. Here we seem to recognize the first "definition" given by Bourbaki at the beginning of his discussion of set theory:

A set is composed of elements capable of having certain properties and having certain relations among themselves or with elements of other sets.

From this ontological point of view the elements, or points, are pre-existent and the problem is to organize them, to give them a structure. From the physical point of view, one postulates with Newton the existence of an absolute space, in which

\footnotetext{
${ }^{12}$ There are two famous incidents: in Nice in September 1970 and at Anvers in July 1972. He alienated a "public mathematical opinion" that was very receptive to his thesis by his brash proclamations and ruined the delicate political arrangements of his friends; he destroyed months of effort in ten minutes.

${ }^{13}$ For a considerable time he practiced dietary restrictions that could be ascribed to choice, Judaism, or Buddhism.
} 
the phenomena occur: positions are predetermined, destined to be inhabited by the accidents of matter.

In Mach's philosophy, on the contrary, space is determined by matter; the most advanced mathematical form is certainly furnished by the Einstein gravitational equations:

$$
R_{\mu \nu}-\frac{1}{2} g_{\mu \nu} R=8 \pi \kappa T_{\mu \nu}
$$

The right-hand side, $T_{\mu \nu}$, is determined by the matter that happens to be present, or, in more modern form, it is a function of the nongravitational fields, while the left-hand side is a function of only the gravitational field $g_{\mu \nu}$, identified with the metric tensor that defines the geometry. In contrast to Newton's views, the space is no longer a mere receptacle, but an actor in physics, as the bending of light rays in a gravitational field shows. For Mach and Einstein, a point then only appears as a label making it possible to identify an event.

A monad has no structure, nor has a point. The same is true of the atom in the original Greek conceptions. It is indivisible and hence cannot reveal any internal mechanism, has no parts, but that does not prevent its having external characteristics, a size certainly, a shape perhaps 14 However, the history of physics has gotten us accustomed to Russian doll games, the chemist's molecule being composed of atoms, which themselves possess an electron cloud and a nucleus, the latter being composed of protons and neutrons, which turned out to be sets of quarks, provisionally assumed to be indivisible. On the mathematical level, the modern view of the continuum presupposes only two levels. A straight line, for example, is made up of pre-existent points. The classical view in the eighteenth century assumes, on the contrary, a hierarchy of infinitesimals and infinities of various orders. To a given order the infinitesimals of the immediately higher order appear to be points without structure, until we open the box that they constitute and that reveals infinitesimals of a higher order playing provisionally the role of points. Brouwer, and to a lesser extent Hermann Weyl, attempted to base a mathematical theory of the continuum on these ideas.

The numerous known axiomatic presentations of geometry encounter similar problems. For Euclid there are geometric figures, and a point is merely one element of a figure, the most elementary, perhaps, since it is assumed to have no dimension: neither length, nor breadth, nor thickness. Figures generate one another. A given line $D$ is defined by the property of passing through two given points $P$ and $Q$, but a point $P$ is defined as the intersection of two lines $D$ and $\Delta$. A circle is defined by giving its center and radius .... There is, to be sure, a determinacy property: two lines, or two circles, that have the same points are equal, but it is not in the spirit of Euclid to regard a line as a set of points. A figure in the sense of Euclid is more than a simple set of points. This traditional point of view is genetic: lines are generated. The modern point of view is ontological: points pre-exist and have no identity of their own; like the monads, they are merely subject to relations. The same kind of duality occurs in formal logic. For the syntactical point of view there are basic formulas and rules for generating new ones, which increase the stock

\footnotetext{
${ }^{14}$ We recall the expression hooked atoms, which has passed into current usage.
} 
of available formulas without limit. In the semantic point of view this potential infinity of formulas is opposed by the convenient fiction of the actual infinity of all the formulas, considered to have been constructed once and for all 15

The theory of lattices was invented by Birkhoff and developed by Glivenko during the 1920s, partly in order to provide an axiom system for projective geometry. In this system linear manifolds of all dimensions are placed on the same level. The fundamental relation is incidence (or inclusion): the straight line $\Delta$ lies in the plane $\Pi$. After that, we obtain the derived concepts of intersection and join (for example, the plane defined by a point and a line). The natural outcome of this direction is furnished by the combinatorial geometries of Rota and Crapo (also called matroids) [4. The algebraization of logic by Boole and Venn (in the nineteenth century) is controlled by the same strategy: the basic given is that of a proposition, or assertion, and implication of propositions plays the role of incidence of linear manifolds.

In order to apply the same methods in topology one must describe a space not by its points but by the class of its open sets, the third example of a lattice. It is in the work of Ehresmann that one finds this point of view explicitly, but Brouwer's ideas, reworked and deepened by Weyl in Das Kontinuum, also lead to it. In reflecting on the well-known problem of the infinity of decimal expansions, Brouwer criticized the possibility of affirming the equality of two numbers 16 but he held that the notion of the open interval $] \frac{1}{4}, \frac{3}{4}$ [ was legitimate, that is, that it is possible to verify the inequalities $\frac{1}{4}<x<\frac{3}{4}$ (if they hold) by a finite process. But the decisive step was taken by Grothendieck. Inspired by Riemann's idea of a surface stacked over the plane 17 he replaced the open sets of a space $X$ by spaces stacked over it. The same thing can be expressed by considering the category $\mathfrak{F}(X)$ of sheaves over $X$. The constructions over topological spaces translate into (and are replaced by) constructions on categories of sheaves. By an additional stage of abstraction Grothendieck, followed by Lawvere and Tierney, proposed an abstract concept of "topos" that was for him the ultimate generalization of the concept of space. But the concept of topos is sufficiently general for the category of "all" sets to constitute a topos. After Cantor and Hilbert, who refused to be driven from Cantor's paradise, it became customary to fit all of mathematics into the framework of the particular topos of sets. Grothendieck claimed the right to transcribe mathematics into any topos whatever. Brouwer and Heyting had long since remarked that the rules of the intuitionistic propositional calculus resemble the rules for manipulation of open sets. This becomes clear in the theory of topos: In any topos $\mathfrak{T}$ there is a logical object $\Omega$, whose "elements" are the truth values of the topos. When $\mathfrak{T}$ is the topos of sets, one has the classical values (true/false), but when the topos $\mathfrak{T}$ is the topos of sheaves over a space $X$, the truth values correspond to the open sets of $X 18$

\footnotetext{
${ }^{15}$ In algebra the potential point of view consists of defining a group or an algebra by generators and relations; the actual point of view consists of defining it as a set of elements structured by operations.

${ }^{16}$ For the verification presumes that one can verify an infinite number of equalities among the decimal digits.

${ }^{17}$ Which gives the definitive interpretation of multi-valued analytic (or holomorphic) functions.

${ }^{18}$ This opens the possibility of a logic in which hic and nunc are considered. An attempt has been made, with J. Bénabou, to provide "topical" foundations for legal logic, especially in a situation involving federal or international law.
} 


\section{TOWARD THE CONCEPT OF SPECTRUM}

Let us provisionally accept Grothendieck's point of view on space. A space $X$ is described using the topos $\mathfrak{F}(X)$ of sheaves over $X$. What is the role of points? If $a$ is a point of $X$, one can first associate with it the "filter" $\mathfrak{U}_{a}$ of open subsets $U$ of $X$ containing $a$, that is, the open neighborhoods of $a 19$ Identifying the point $a$ with the filter $\mathfrak{U}_{a}$ leads to a fruitful strategy. To enrich the space $X$, we introduce ideal points in correspondence with other filters. It is in this way that Bourbaki constructs the completion of a uniform space and that various compactifications (such as the Stone-Cech compactification) are defined, as well as various boundaries in potential theory or in the theory of deterministic or stochastic dynamic systems.

A similar point of view prevails in the theory of models in logic. A model $M$ of a set $\mathcal{P}$ of propositions has the effect of validating certain propositions $A$, which one denotes as

$$
M \vdash A .
$$

Instead of the model $M$, one may legitimately consider the class $\mathcal{P}_{M}$ of propositions validated by $M$, which is also a filter 20 The model is consistent if not all propositions are valid in it. The same thing can be expressed by saying that the model $M$ does not validate simultaneously a proposition $A$ and its negation $\bar{A}$. In contrast, the model $M$ is categorical if for each proposition $A$ it validates either $A$ or $\bar{A}$. In a categorical model one can then introduce the valuation $v(A)$ (or, more precisely, $v_{M}(A)$ ), which has the value 1 if $M$ validates $A$ and 0 otherwise. We have the following algebraic properties:

$$
\begin{aligned}
v(A)+v(B) & =v(A \wedge B)+v(A \vee B) \\
v(T) & =1, \quad v(F)=0
\end{aligned}
$$

which can be taken as the definition of a valuation 21 This concept admits several variants:

1. a valuation on the set of open sets of a space $X$ corresponds to an ultrafilter (of open sets) over $X$;

2. instead of requiring the valuation $v(A)$ of a proposition to assume only the values 0 and 1 , one may postulate more generally that $v(A)$ is a real number between 0 and 1 . This is roughly the strategy of "fuzzy" logic 22

We shall now apply a trick that is one of the spectacular successes of twentiethcentury mathematics. Let us again consider an algebra $\mathcal{P}$ of propositions 23 and the set $\Omega$ of all valuations on $\mathcal{P}$. With each proposition $A$ we associate by duality the set $[A]$ of all valuations $v$ such that $v(A)=1$, or, what amounts to the same thing, the set of all categorical models that validate $A$. The correspondence $A \rightleftarrows[A]$ makes it possible to interpret the algebra of propositions $\mathcal{P}$ as a class of subsets of

\footnotetext{
${ }^{19}$ The filter property of $\mathfrak{U}_{a}$ means the following: If $U$ and $V$ belong to $\mathfrak{U}_{a}$, so do the intersection $U \cap V$ and all open sets $U^{\prime}$ containing $U$.

${ }^{20}$ This means that, if the propositions $A$ and $B$ are in $\mathcal{P}_{M}$, the same is true of the conjunction $A \wedge B(\operatorname{read} A$ and $B)$ and any proposition $A^{\prime}$ implied by $A$.

${ }^{21}$ Here $T(F)$ denotes any true (false) proposition.

${ }^{22}$ Carathéodory and Kappos used this method to construct an alternative to the axiomatization of probability due to Kolmogorov. Despite the philosophical interest of this method, it is technically more cumbersome than Kolmogorov's approach, especially for the study of stochastic processes.

${ }^{23}$ More precisely, a Boolean algebra.
} 
$\Omega$, conjunction and disjunction becoming respectively the intersection and union of sets:

$$
[A \wedge B]=[A] \cap[B], \quad[A \vee B]=[A] \cup[B] .
$$

This is the statement of the Stone representation theorem, a model for many further results 24 The space $\Omega$ is called the Stone space of the Boolean algebra $\mathcal{P}$; we shall call it, somewhat anachronistically, the spectrum of $\mathcal{P}$.

The development of quantum theory during the 1930s and 1940s led to the concept of a state, which is a new embodiment of the concept of a valuation. From now on we assume that the space $X$ is compact 25 and associate with it the Banach space $A=C^{0}(X ; \mathbb{C})$ formed of continuous functions with complex values on $X$, with the norm

$$
\|f\|=\sup \{|f(a)|: a \in X\} .
$$

One can also introduce the product $f_{1} f_{2}$ of two functions and the complex conjugate $f^{*}$ of $f$. A state on $A$ is a linear form $\eta$ on $A$ satisfying the following rules:26

$$
\eta\left(f f^{*}\right) \geq 0, \quad \eta(\mathbf{1})=1 .
$$

A state $\eta$ is pure if it is never the mixture $\eta=\frac{1}{2}\left(\eta_{1}+\eta_{2}\right)$ of two distinct states. The Gelfand representation theorem asserts that the formula

$$
f(a)=\eta(f) \text { for every } f \text { in } A
$$

defines a one-to-one correspondence between the points $a$ of $X$ and the pure states $\eta$ of the Banach algebra $A$. One thus has a means of recovering the space $X$ using the Banach algebra $A$.

The concept of a filter of a Boolean algebra is the analog of the concept of an ideal in a commutative Banach algebra. In both cases there is an inclusion relation and hence the concept of a maximal filter or ideal 27 The spectrum of the Boolean algebra $\mathcal{P}$ (or the Banach algebra $A=C^{0}(X ; \mathbb{C})$ ) can then be interpreted as the set of maximal filters (or ideals). In number theory the idea of considering the set of prime ideals in the ring $\mathfrak{O}_{K}$ of algebraic integers in a number field $K$ (a finite algebraic extension of the field $\mathbb{Q}$ of rational numbers) is due to Dedekind. For him this set is the arithmetic analog of the set of points of an algebraic curve. Great effort was made during the 1950s to provide the broadest possible foundations to algebraic geometry. The following are two important stages:

1. Consider an algebraically closed field $k$ and an algebraic subvariety $X$ of the affine space $k^{n}$ of coordinates $T_{1}, \ldots, T_{n}$. Let $\mathfrak{a}$ be the ideal of polynomials that vanish identically on $X$, and let $A$ be the quotient ring $k\left[T_{1}, \ldots, T_{n}\right] / \mathfrak{a}$. The intrinsic geometric properties of $X$ (independent of the immersion of $X$ in $k^{n}$ ) translate into properties of the ring $A$. In particular, the points of $X$ correspond to maximal ideals of $A$. (This is the content of the Hilbert Nullstellensatz.) The analogy with the Gelfand representation theorem is obvious; it is one of the points of departure of Serre's theory of algebraic coherent sheaves [11].

${ }^{24}$ This result also shows a posteriori that the point of view of Carathéodory and Kappos on probability is not more general than that of Kolmogorov.

${ }^{25}$ The case when $X$ is locally compact makes certain minor changes necessary.

${ }^{26}$ The function having the constant value 1 on $X$ is denoted $\mathbf{1}$.

${ }^{27}$ Maximal filters are usually called ultrafilters. 
2. Consider now an affine or projective algebraic variety $X{ }^{28}$ defined over a field $k$. The field $k$ is not assumed algebraically closed, but $X$ is irreducible. One can define the field $K=k(X)$ of rational functions on $X$ and, for each point $x$ of $X$, the ring $\mathfrak{O}_{x}$ of rational functions defined at the point $x$. More generally, if $Y$ is an irreducible subvariety of $X$, we define the local ring of $X$ along $Y$, denoted $\mathfrak{O}_{X, Y}$. Zariski had developed the theory of these local rings, but it is Chevalley who, in his seminar 2] of 1956, made the collection of $\mathfrak{O}_{X, Y}$, which he called the scheme of $X$ (for variable $Y$ ), the basis of the theory. He gave an axiomatic characterization of these schemes.

It was necessary to synthesize these points of view and to eliminate the preceding restrictions (where $k$ is algebraically closed or $X$ is irreducible). After various preliminary attempt: 29 Grothendieck understood how to define the general notion of scheme using the Zariski topology 30 and a sheaf of local rings. One must, of course, understand that the space Grothendieck associated with an algebraic variety is not the set of its own points, but the set of its irreducible subvarieties. That is the meaning of the word scheme! As for the points, see the next section.

Now one final remark on the term spectrum. In physics each type of atom or molecule possesses a characteristic spectrum formed by its emission or absorption lines. Quantum mechanics interprets these as the characteristic values of an operator, the Hamiltonian, acting on a certain Hilbert space. It is thus natural to speak of the discrete spectrum of the Hamiltonian. The emission or absorption bands correspond to a continuous spectrum. In the early 1930s von Neumann succeeded brilliantly in defining the concept of a self-adjoint (unbounded) operator $H$ on a Hilbert space $\mathfrak{h}$ and its spectrums. The contribution of Gelfand in 1940 was in associating a commutative Banach algebra $A$ with the operator $H$ and an isomorphism of $A$ onto $C^{0}(S ; \mathbb{C})$. From that point on the evolution of the meaning of the word spectrum can be understood. For Grothendieck the spectrum of a commutative ring consists of its prime ideals (as in the case of Dedekind).

\section{Points and Representations}

If all points are intrinsically indistinguishable from one another, they can differ only in position. In other words, there exists an archetypal point, of which the other points are representations. Let us translate this idea into more mathematical language. We introduce a space consisting of a single point 1 . For each point $a$ of a space $X$ there exists a unique mapping, or representation, of the space 1 into the space $X$ mapping the one point of $\mathbf{1}$ to the point $a$ of $X$. Category theory is the mathematical expression of the idea of representation (or transformation). We have a class of objects (or spaces) and transformations $f$ of an object $X$ into an object

\footnotetext{
${ }^{28}$ Or even an abstract variety in the sense of Weil.

${ }^{29}$ Serre first considered the set of maximal ideals of a commutative ring $A$ subject to certain restrictions. Martineau then remarked to him that his arguments remained valid for any commutative ring, provided one takes all prime ideals instead of only maximal ideals. I then proposed a definition of schemes equivalent to the definition of Grothendieck. In my dissertation I confined myself to a framework similar to that of Chevalley, so as to avoid an excessively long exposition of the preliminaries!

${ }^{30}$ In the case of Chevalley one has a collection $\left(V_{s}\right)_{s \in S}$ of local rings having the same field of fractions $K$. A subset $U$ of $S$ is open in the Zariski topology if there is a subset $F$ of $K$ such that $s \in U$ if and only if $F \cap V_{s}$ is nonempty. One then defines $\mathfrak{O}(U)=\underset{s \in U}{\cap} V_{s}$, and this collection of rings defines the structure sheaf over $S$.
} 
$Y$, with the possibility of composing these transformations. The "idea" of category theory is to consider only spaces and transformations rather than points. However, in the majority of categories there exists an object such as $\mathbf{1}$ characterized by the fact that there is exactly one transformation from $X$ into $\mathbf{1}$, whatever the object $X$. One can thus call any transformation of 1 into $X$ a point of $X$, but it may very well happen that an object has no points in this sense.31

Let us examine the case of Boolean algebras. We take as basic operations in a Boolean algebra $\mathcal{P}$ the conjunction $A \wedge B$ and the disjunction $A \vee B$. The comparison relation $A \leq B$ is indeed synonymous with $A=A \wedge B$ and also with $B=A \vee B$. A representation $f$ of one Boolean algebra $\mathcal{P}$ into another $\mathcal{P}^{\prime}$ is a mapping $f$ of $\mathcal{P}$ into $\mathcal{P}^{\prime}$ that obeys the rules 32

$$
f(A \vee B)=f(A) \vee f(B), \quad f(A \wedge B)=f(A) \wedge f(B) .
$$

There exists a Boolean algebra, denoted $\mathbf{1}$, which is composed of two elements 0 and 1 with the rules 33

$$
\begin{aligned}
& 0 \wedge 0=0 \wedge 1=1 \wedge 0=0 \quad 1 \wedge 1=1 \\
& 1 \vee 1=0 \vee 1=1 \vee 0=1 \quad 0 \vee 0=0
\end{aligned}
$$

from which follows $0 \leq 1$. For every Boolean algebra $\mathcal{P}$ there is one and only one representation of $\mathbf{1}$ in $\mathcal{P}$, and the valuations of $\mathcal{P}$ are the representations of $\mathcal{P}$ into 1. To conform to the general scheme described above, we use the procedure of dualization or "arrow reversal". We call a "representation" of $\mathcal{P}$ into $\mathcal{P}^{\prime}$ a "noitatneserper" of $\mathcal{P}^{\prime}$ into $\mathcal{P}$. The spectrum of $\mathcal{P}$ is then the set of noitatneserpers of 1 into $\mathcal{P}$.

The necessity of reversing the sense of the arrows shows up more clearly in the case of the Gelfand representation. If $X$ and $Y$ are compact spaces, every continuous mapping $u$ of $X$ into $Y$ defines in the inverse sense a representation $u^{*}$ of the algebra $B=C^{0}(Y ; \mathbb{C})$ into the algebra $A=C^{0}(X ; \mathbb{C})$. Conversely, every representation $\Phi 34$ of the algebra $B$ into the algebra $A$ arises from a unique continuous mapping $u$ from $X$ into $Y$ via the formula $\Phi=u^{*}$. Corresponding to the one-point space is the algebra $\mathbb{C}$ of complex numbers, and the space $X$ can be interpreted as the set of representations of the algebra $A=C^{0}(X ; \mathbb{C})$ into the algebra $\mathbb{C}$. More generally, if $A$ is a commutative Banach algebra with unit $\mathbf{1}$, the pure states of $A$ are the representations of $A$ into $\mathbb{C}$, which gives a new interpretation of the spectrum of $A$.

Algebraic geometry had long encountered the problem of equations having no solutions, that is, spaces having no points. Let us consider for example an imaginary circle in the plane; one may assume that its equation is written as

$$
x^{2}+y^{2}+a^{2}=0 .
$$

To be sure, if the real number $a \neq 0$ is given, there is no point with real coordinates $x$ and $y$ satisfying (9). The complex numbers were invented to provide solutions to such equations. In Grothendieck's point of view, one first introduces the quotient

\footnotetext{
${ }^{31}$ For example, in the category of bundles over a fixed base $S$ the "points" of a bundle $E$ are global sections. Many interesting bundles have no such section. The Hopf bundle (the sphere $\mathbb{S}^{3}$ of dimension 3 , fibered over $\mathbb{S}^{2}$ with fibers homemorphic to $\mathbb{S}^{1}$ ) has none. For physicists the Hopf bundle is the Dirac monopole.

${ }^{32}$ The relation (4) can thus be interpreted in terms of representation of Boolean algebras.

${ }^{33}$ If we interpret 1 in the usual manner as the value "true" and 0 as "false", this Boolean algebra gives universal rules for the manipulation of true and false.

${ }^{34}$ In other words, $\Phi$ is a linear mapping of $B$ into $A$ such that $\Phi\left(b b^{\prime}\right)=\Phi(b) \Phi\left(b^{\prime}\right)$ and $\Phi\left(\mathbf{1}_{B}\right)=$ $\mathbf{1}_{A}$, denoting by $\mathbf{1}_{A}$ the identity element of $A$ and by $\mathbf{1}_{B}$ the identity element of $B$.
} 
algebra $\mathfrak{O}=\mathbb{R}[X, Y] /\left(X^{2}+Y^{2}+a^{2}\right)$. The circle $\Gamma$ of (9) is then the spectrum of the ring $\mathfrak{O}$, that is the set of its prime ideals. The complex solutions of (9), in other words the complex points of the circle $\Gamma$, correspond to the $\mathbb{R}$-linear representations of $\mathfrak{O}$ into $\mathbb{C}$. This suggests that we introduce, for each commutative algebra $A$ over $\mathbb{R}$, the set $\Gamma(A)$ of $\mathbb{R}$-linear representations of $\mathfrak{O}$ into $A$, which we shall call the $A$-points of $\Gamma$. The "real" circle $\Gamma$ (that is, the circle whose equation has real coefficients) has no $\mathbb{R}$-points, but it has plenty of $\mathbb{C}$-points. In the case of an arbitrary scheme, the consideration of the $A$-points for all commutative algebras makes it possible to invoke a familiar situation 35 For example, if $G$ is a group scheme, the $A$-points form a group $G(A)$, in the standard sense, for any $A$.

Consideration of the topos $\mathfrak{F}(X)$ of sheaves on a topological space $X$ makes it possible, following Grothendieck, to give another extension of the notion of a point. If $X$ and $Y$ are two topological spaces and $u$ a continuous mapping of $X$ into $Y$, we associate with $u$ a covariant functor $u_{*}$ and a contravariant functor $u^{*}$. If $F$ is a sheaf over $X$ and $G$ a sheaf over $Y$, then $u_{*} F$ is a sheaf over $Y$ and $u^{*} G$ a sheaf over $X$. One can easily describe $u_{*} F$ by the formula

$$
u_{*} F(V)=F\left(u^{-1}(V)\right)
$$

for any open set $V$ in $Y$. Moreover, the functors $u_{*}$ and $u^{*}$ are adjoints, which means that the representations $F \rightarrow u^{*} G$ of sheaves over $X$ are in one-to-one correspondence with the representations $u_{*} F \rightarrow G$ of sheaves over $Y$. This property contains an implicit characterization of the functor $u^{*}$.

The topos $\mathfrak{S e t}$ of sets can be regarded as the topos of sheaves over a space reduced to a point. According to what has just been said, the choice of a point $a$ of a topological space $X$ therefore defines two adjoint functors:

$$
\mathfrak{F}(X) \underset{a_{*}}{\stackrel{a^{*}}{\rightleftarrows}} \mathfrak{S e t} .
$$

The functor $a^{*}$ associates with each sheaf over $X$ its fiber 36 at the point $a$. This circumstance explains why Grothendieck and Deligne spoke interchangeably of "point" or "fiber functor".

\section{TOWARD NON-COMMUTATIVE GEOMETRY}

We continue to pursue the theme of spaces without points, or with too few points. Consider, for example, a group $G$, assumed discrete, operating on a space * reduced to a single point. What can be said about the space of orbits of $G$ in the space $*$ ?

\footnotetext{
${ }^{35}$ This idea actually goes back to André Weil [13], who introduced it into differential geometry. For example, if $A$ has base $1, \varepsilon$ over $\mathbb{R}$ with $\varepsilon^{2}=0$, the $A$-points of a variety $X$ are the points of the tangent bundle $T X$ to $X$. More generally, the spaces of jets are handled in this way. In my 1958 dissertation I introduced a similar idea for algebraic groups, which made it possible for me to handle efficiently what are known as inseparable isogenies. The question involves representations $f: G \rightarrow H$ of algebraic groups that are bijections or points, yet not isomorphisms. By point I mean the $k$-points, where $k$ is the base field assumed to be algebraically closed and of characteristic $p>0$.

${ }^{36}$ By definition it is the inductive limit of the sets $F(U)$ when $U$ ranges over all open neighborhoods of $a$.
} 
At first sight there is only one orbit, and the group $G$ has disappeared. However, three more subtle answers are known:

1. According to Armand Borel [1, one should not distinguish two homotopically equivalent spaces. The space $*$ is therefore replaced by a contractible space $E G$ on which $G$ operates freely. The space of orbits of $G$ in $*$ is then interpreted as the base $B G=E G / G$ of $E G$ considered as a principal bundle with group $G$. More generally, if the group $G$ operates on a space $X$, the "true" orbit space, denoted $X / / G$, is the quotient of $X \times E G$ by the diagonal action of $G$. What has just been described is the classifying space method.

2. According to Grothendieck $\left[\mathrm{T}\right.$ one considers the topos $\mathfrak{E}_{G}$ of sets on which the group $G$ operates. These $G$-sets are to be interpreted as sheaves over the mythical space of orbits of $G$ in $*$. In view of the preceding construction, one may call $\mathfrak{E}_{G}$ the classifying topos of $G$, since $B G$ is called the classifying space of $G$. More generally, let us assume that the group $G$ operates continuously on a topological space $X$. One can define the naive space of orbits $X / G$, but it may happen that the space $X / G$ is pathological and has only "trivial" open sets (the entire space and the empty set) 37 In this case the information provided by the sheaves over $X / G$ will treat this space as a single point. In that case Grothendieck proposed replacing the sheaves over $X / G$ by the sheaves $F$ over $X$, with the compatible action of $G$ over $X$ and $F$. The topos $\mathfrak{F}(X / G)$ is replaced by the topos $\mathfrak{F}(X ; G)$ of $G$-equivariant sheaves over $X$.

The classifying space $B G$ has homology groups, which are precisely the homology groups of the group $G$ in the algebraic sense. The sheaf cohomology techniques have a generalization to topos. Applied to the topos $\mathfrak{F}(X ; G)$, they provide the definition of equivariant cohomology groups for the $G$-space $X$. They can be interpreted as cohomology groups for the nonexistent space $X / G$.

3. We shall now describe the method of Alain Connes [NC]. On a discrete space $X$ having a finite number of points, a bundle $F$ of vector spaces over $X$ is precisely the set of fibers $\left(F_{a}\right)_{a \in X}$, and the sections of $F$ form the vector space $\Gamma(F)=\underset{a \in X}{\oplus} F_{a}$. The algebra $\mathbb{C}^{X}$ of functions on $X$ operates on this space by multiplication. In Hilbertian analysis, we thus have the following translations:

(a) a bundle over a locally compact space $X$ corresponds to an involutive representation 38 of the algebra $C_{\infty}^{0}(X ; \mathbb{C})$ of continuous functions on $X$ that vanish at infinity;

(b) a group action 39 of $G$ corresponds to a unitary representation of the group $G$ in a Hilbert space.

\footnotetext{
${ }^{37}$ As a simple example, $X$ is a circle and $G$ consists of the rotations through an angle $n \theta$, where $n$ is an arbitrary integer and $\theta / 2 \pi$ is irrational.

${ }^{38}$ If $A$ is a $C^{*}$-algebra and $\mathfrak{h}$ a Hilbert space, an involutive representation of $A$ in $\mathfrak{h}$ is a linear mapping $\pi: A \rightarrow \mathcal{L}(\mathfrak{h})$ such that$$
\pi(S T)=\pi(S) \pi(T), \quad \pi(1)=1, \quad \pi\left(S^{*}\right)=\pi(S)^{*} .
$$

The set of bounded linear operators on $\mathfrak{h}$ is denoted $\mathcal{L}(\mathfrak{h})$.

${ }^{39}$ The group $G$ is assumed locally compact. In particular, it may be discrete. 
To each locally compact group $G$, as is known, one can associate 40 a $C^{*}$-algebra $C^{*}(G)$ such that the unitary representations of $G$ correspond to the involutive representations of $C^{*}(G)$. In Connes' perspective this algebra $C^{*}(G)$ corresponds to the classifying space $B G$. When the group $G$ is commutative with Pontryagin dual $\widehat{G}$, the $C^{*}$-algebras $C^{*}(G)$ and $C_{\infty}^{0}(\widehat{G} ; \mathbb{C})$ are isomorphic. One would thus expect some close connections between $B G$ and $\widehat{G}$, and such is indeed the case if $G=\mathbb{Z}^{n}$, whence $B G=\widehat{G}=U(1)^{n}$. It should be noted, nevertheless, that $B G$ is defined only up to a homotopy, while $\widehat{G}$ is defined uniquely.

Returning to the case of a group $G$ acting on a space $X$, we find that the analog of a $G$-equivariant bundle (or sheaf ) over $X$ is provided by a compatible pair of representations, one of $C_{\infty}^{0}(X ; \mathbb{C})$, the other of $G$, on the same Hilbert space. It is known how to define a $C^{*}$-algebra $C^{*}(X ; G)$ whose involutive representations correspond to the pairs described above. This algebra plays the role of the algebra of continuous functions on the space of orbits of $G$ in $X$.

One can handle the case of foliations similarly. We summarize the situation in the following diagram:

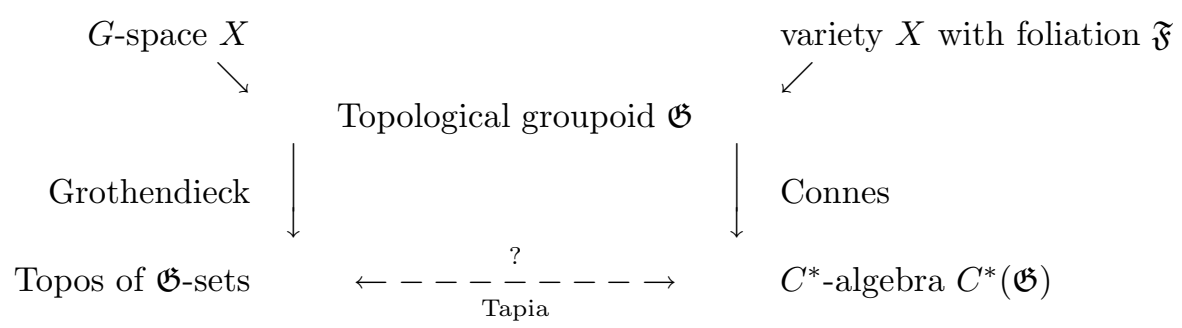

The two theories are unified by the groupoid concept. Groupoids are generalizations of groups, and a considerable portion of the machinery of groups extends to them. Hence, in particular, we have the notion of $\mathfrak{G}$-set and the $C^{*}$-algebra $C^{*}(\mathfrak{G})$ (if $\mathfrak{G}$ is locally compact). The $C^{*}$-algebra $C^{*}(X ; G)$ is precisely the $C^{*}$-algebra $C^{*}(\mathfrak{G})$, where $\mathfrak{G}$ is a locally compact groupoid constructed from the action of $G$ on $X$. The other cases are similar.

We have the following natural concepts of equivalence:

1. homotopy, for topological spaces;

2. topos equivalence;

3. Morita equivalence for $C^{*}$-algebras.

The "topological" invariants must be compatible with these equivalences. The "pathological" character of the action of a group or foliation is translated as the fact that the associated topos (or $C^{*}$-algebra) is not equivalent to that of an ordinary space. In the case of a $C^{*}$-algebra, this is translated as the fact that it is not equivalent in the sense of Morita to a commutative $C^{*}$-algebra, in other words, that it is essentially noncommutative. One can now understand the sense of the expression "noncommutative geometry". A comparison between the points of view

\footnotetext{
${ }^{40}$ The space of functions on $G$ that are integrable in the sense of Lebesgue with respect to Haar measure is denoted $L^{1}(G)$. This space is a Banach algebra with convolution as the multiplication. A state $\eta$ on $L^{1}(G)$ is a continuous linear functional of norm 1 on $L^{1}(G)$ such that $\eta\left(f * f^{*}\right) \geq 0$ for any $f \in L^{1}$. The spectral norm is defined as the least upper bound $\|f\|_{S}$ of the numbers $\eta\left(f * f^{*}\right)^{1 / 2}$ as $\eta$ ranges over the set of states. The algebra $C^{*}(G)$ is then obtained as the completion of $L^{1}(G)$ in the spectral norm.
} 
of Grothendieck and Connes has barely been sketched out (see the paper [12] of J. Tapia).

What are the points of a generalized space? We defined the points of a topos in Section 5. The points of a $C^{*}$-algebra are equivalence classes of irreducible involutive representations 41

\section{INTERNAL SYMMETRIES}

We begin to suspect that not all points are alike - there are several species of monads. The question thus arises of finding out whether a point can have symmetries. Let us consider the simple case of a finite group $G$ operating on a compact manifold $X$ and the naive space of orbits $X / G$. If $\xi$ is the orbit $G \cdot x$ of a point $x$ of $X$, there are numerous reasons to consider that the stabilizer $42 G_{x}$ of $x$ in $G$ is the symmetry group of the point $\xi$ of $X / G$. In particular, the naive point of */G (see Section [6) has $G$ as its symmetry group. That explains the inadequacy of the point of view of sheaves over the naive quotient $X / G$. The peculiarity of a sheaf is that what is known at a point is known unambiguously at all sufficiently near points, and that excludes the possibility of branching 43 These simple remarks were developed in Satake's theory of $V$-manifolds (called "orbifolds" by Thurston), which have found numerous applications in mathematical physics.

The question of internal symmetries is very acute in elementary particle physics. The Kaluza-Klein model was introduced in the 1920s in an attempt to unify electromagnetism and gravitation. By way of illustration, let us first consider the surface of a circular cylinder with axis $D$ and radius $r$ (the set of points in 3-dimensional space lying at distance $r$ from the line $D$ ). Every plane perpendicular to the axis cuts the cylinder in a circle of radius $r$, and the cylinder is the union of these pairwise-disjoint circles. If the radius $r$ is very small, each of the circles can be identified with its center, and the cylinder becomes indistinguishable from its axis. The transversal dimension has become compactified. To be sure, each circle has its rotation group $\Gamma$, and this group is not to be forgotten when the circle is contracted to its center. One can thus introduce $\Gamma$ as the group of internal symmetries of each point of the line $D$ by adjoining an additional dimension. In more technical terms, the cylinder appears as the principal bundle with base $D$ and group $\Gamma$. Returning to the Kaluza-Klein model, we replace the 4-dimensional space-time $M^{4}$ by a 5 -dimensional space by blowing up each point of $M^{4}$ into a "very small" circle. The group of internal symmetries thus associated with each point is the preceding group $\Gamma=\mathrm{SO}(2)$, which appears implicitly in the change of gauge of electromagnetic potential 44

\footnotetext{
${ }^{41}$ By the Gelfand-Naimark-Segal theorem, for any pure state $\eta$ on the $C^{*}$-algebra $A$ there exists an irreducible involutive representation $\pi_{\eta}: A \rightarrow \mathcal{L}\left(\mathfrak{h}_{\eta}\right)$ and a vector $\psi_{\eta}$ in the Hilbert space $\mathfrak{h}_{\eta}$ such that $\eta(S)=\left\langle\psi_{\eta}\left|\pi_{\eta}(S)\right| \psi_{\eta}\right\rangle$ for $S$ in $A$. We introduce an equivalence relation on the set of pure states by setting $\eta \equiv \eta^{\prime}$ if $\pi_{\eta}$ is equivalent to $\pi_{\eta^{\prime}}$. Then a point of $A$ is an equivalence class of pure states.

${ }^{42}$ The set of elements $g$ of $G$ such that $g \cdot x=x$.

${ }^{43}$ The phenomenon of branching is well known for analytic functions of a complex variable. At a branch point $z_{0}$ of order $n$ it is necessary to use power series in $\left(z-z_{0}\right)^{\frac{1}{n}}$, but this $n$th root has $n$ branches near $z_{0}$. These $n$ branches permute cyclically among themselves, whence arises the idea of a cyclic group at the point $z_{0}$ whose order $n$ is the order of the branching.

${ }^{44}$ Denoting the components of electromagnetic potential by $A_{\mu}$, we find that the components of the electromagnetic field are $F_{\mu \nu}=\partial_{\mu} A_{\nu}-\partial_{\nu} A_{\mu}$. The indices $\mu$ and $\nu$ assume the values $0,1,2,3$ corresponding to coordinates $x^{0}, x^{1}, x^{2}, x^{3}$ in space-time, and we have used the notation
} 
In an attempt to take account of other fields besides the electromagnetic field and other particles besides electrons and photons, the same strategy was applied, with the group $\mathrm{SO}(2)$ (isomorphic to $\mathrm{U}(1)$ ) being replaced by the groups $\mathrm{SU}(2)$ and $\mathrm{SU}(3)$, or even more complicated groups such as $E_{8}$ in certain models. The 4-dimensional space $M^{4}$ is replaced by a space of $4+d$ dimensions, where $d$ is the dimension of the group. In the 1960 s an attempt was made to unify the Poincaré group of external symmetries of space-time with the group of internal symmetries. This unification was thwarted for mathematical reasons 45

Grothendieck's point of view makes it possible to introduce the symmetry group of a point. The fundamental remark is the following: suppose one is given two categories $\mathcal{C}$ and $\mathcal{C}$ '. One can define transformations of categories (called "functors"), say $T: \mathcal{C} \rightarrow \mathcal{C}^{\prime}$, and also transformations between functors according to the following scheme:

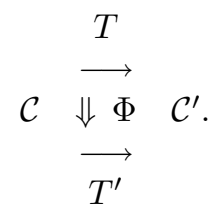

Functors can be composed, as can transformations between functors. One can therefore speak of the automorphism group of a functor, $T: \mathcal{C} \rightarrow \mathcal{C}^{\prime}$, namely the transformations $T \stackrel{\Phi}{\Rightarrow} T$ that have an inverse. Since a point in the sense of Grothendieck is interpreted as a functor between topos, the trick has worked: the point has a symmetry group. For example, if $X$ is a topological space and $a$ a point of $X$, Grothendieck defines the fundamental group $\pi_{1}(X ; a)$ of $X$ at the point $a$ as the automorphism group of the fiber functor $a^{*}$ restricted to the subtopos $\mathfrak{F}_{l c}(X)$ of locally constant sheaves over $X$

$$
a^{*}: \mathfrak{F}_{l c}(X) \rightarrow \mathfrak{S e t}
$$

In other words, the fundamental group $\pi_{1}(X ; a)$ is regarded as the symmetry group of the point $a$. Thanks to the introduction of the spectrum of a field, the Galois group $\mathrm{Gal}(K / k)$ of a field extension can also be interpreted as the symmetry group of a point!

The same idea can be applied to reinterpret the Tannaka-Krein duality theorem. Let $G$ be a compact group. We can introduce the category $\mathfrak{R e p}_{G}$ whose objects are continuous finite-dimensional linear representations of the group $G 46$ We also introduce the category $\mathfrak{V} \mathfrak{c c t}_{f}$ formed of complex finite-dimensional vector spaces and linear transformations. The "fiber" functor $\Phi: \mathfrak{R e p}_{G} \rightarrow \mathfrak{V e c t}_{f}$ associates with a representation $\pi$ the vector space $V_{\pi}$ on which it acts. The group $G$ can

$\partial_{\mu}=\partial / \partial x^{\mu}$. A change of gauge amounts to replacing $A_{\mu}$ by $A_{\mu}+\partial_{\mu} \Phi$, which makes no change in the $F_{\mu \nu}$. But prescribing $\Phi$ defines at each point $x$ of $M^{4}$ a rotation through angle $e \Phi(x) / \hbar$ of the circle expressing the internal symmetry of $x$. We remind the reader that $e$ denotes the elementary electric charge, and $\hbar=h / 2 \pi$, where $h$ is the Planck constant.

${ }^{45}$ By classical theorems of Élie Cartan, one cannot construct any group that is a genuine extension of the external group by the internal group. The best one can do is to form the direct product, that is, to forbid them to interact.

${ }^{46}$ Let $\pi$ be a representation of $G$ on the space $V_{\pi}$ and $\pi^{\prime}$ on the space $V_{\pi^{\prime}}$. A transformation of $\pi$ into $\pi^{\prime}$ is a linear mapping $T$ of $V_{\pi}$ into $V_{\pi^{\prime}}$ such that $T \pi(g)=\pi^{\prime}(g) T$ for every element $g$ of $G$. 
be recovered as the automorphism group of this functor $\Phi$ that preserves the tensor product in a suitable sense 47

The interpretation of this theorem is that a group $G$ exists only by virtue of its category of representations $\mathfrak{R e p} \mathfrak{p}_{G}$. Indeed, the use of groups to classify elementary particles is analogous. In the chain of broken symmetries for hadrons

$$
\mathrm{SU}(3) \supset \mathrm{SU}(2) \supset \mathrm{U}(1) \text {, }
$$

what matters are only the representations of these groups and their branching rules 48 to organize the families of hadrons into multiplets; then separate the members of the multiplet by their electric charge (the group $U(1)$ corresponding to electromagnetism, as explained above).

The problem thus arises of giving an axiomatic characterization of categories of the form $\mathfrak{R e p}_{G}$. Such a characterization was obtained independently by Deligne in the Grothendieck Festschrift [5], as an extension of Grothendieck's program, and by the physicists Doplicher and Roberts [6], who were motivated by the theory of gauge fields.

\section{8. "I HAVE A DREAM"}

Grothendieck's broken dream was to develop a theory of motives, which would in particular unify Galois theory and topology. At the moment we have only odd bits of this theory, but I would like to conclude with a magnificent, quite unexpected development, in which physics and mathematics come together again.

Euler was the first to study the series $\zeta(k)=\sum_{n=1}^{\infty} n^{-k}$ for $k=2,3, \ldots$. In particular, the formulas $\zeta(2)=\pi^{2} / 6$ and $\zeta(4)=\pi^{4} / 90$ are due to him. He was the first to consider multiple series of the same type (the Euler-Zagier numbers [14]):

$$
\zeta\left(k_{1}, \ldots, k_{r}\right)=\sum n_{1}^{-k_{1}} \cdots n_{r}^{-k_{r}}
$$

where the summation extends over all integers $n_{1}, \ldots, n_{r}$ such that $n_{1}>n_{2}>$ $\cdots n_{r} \geq 1$. The product of two such numbers is a linear combination of numbers of the same type. We quote the first interesting case:

$$
\zeta(a) \zeta(b)=\zeta(a+b)+\zeta(a, b)+\zeta(b, a)
$$

which can be proved by elementary manipulations of series. It is conjectured that all polynomial relations with rational coefficients among the numbers $\zeta\left(k_{1}, \ldots, k_{r}\right)$ can be derived from these product relations and others of the same type, known explicitly. To give an idea of the magnitude of this conjecture, I note that it implies that the numbers $\zeta(3), \zeta(5), \ldots$, are transcendental. For a long time it was only known that $\zeta(3)$ is irrational (Apéry, 1979), but Rivoal has recently extended this result by proving that infinitely many numbers in the sequence $\zeta(3), \zeta(5), \ldots$, are irrational $[\mathrm{R}]$.

\footnotetext{
${ }^{47}$ An automorphism of the functor $\Phi$ is defined by giving, for each representation $\pi$ of $G$, an invertible linear operator $S_{\pi}$ in the space $V_{\pi}$ on which $\pi$ acts. We impose the rule $T S_{\pi}=S_{\pi^{\prime}} T$ for every transformation $T$ of $\pi$ into $\pi^{\prime}$. Compatibility with the tensor product is expressed by the formula $S_{\pi \otimes \pi^{\prime}}=S_{\pi} \otimes S_{\pi^{\prime}}$. The duality theorem then states that if $S_{\pi}$ satisfies these conditions, there exists a unique element $g$ of $G$ such that $S_{\pi}=\pi(g)$ for every representation $\pi$.

${ }^{48}$ Which describe how an irreducible representation of a group decomposes into irreducible representations of a subgroup after the representation of the group has been restricted to the subgroup.
} 
We denote by $\mathfrak{A}_{d}$ the set of linear combinations of the numbers $\zeta\left(k_{1}, \ldots, k_{r}\right)$ with rational coefficients, with $k_{1}+\cdots+k_{r}$ equal to $d$. The product formulas show that we have

$$
\mathfrak{A}_{d} \cdot \mathfrak{A}_{d^{\prime}} \subset \mathfrak{A}_{d+d^{\prime}},
$$

so that the direct sum $\mathfrak{A}$ of $\mathfrak{A}_{0}=\mathbb{Q}, \mathfrak{A}_{1}, \mathfrak{A}_{2}, \ldots$, is a commutative algebra over the field $\mathbb{Q}$ of rational numbers. Drinfeld has introduced a group $G R T_{1}$ called the (graded) Grothendieck-Teichmüller group. It is a scheme of groups over the field $\mathbb{Q}$, and it therefore has a Lie algebra, denoted $\mathfrak{g r t}_{1}$. To describe this Lie algebra would require me to give precise information on the Knizhnik-Zamolodchikov equations, which play a fundamental role in the theory of conformal fields. It is conjectured that the Lie algebra $\mathfrak{g r t}_{1}$ is a free Lie algebra with generators $\psi_{3}, \psi_{5}, \psi_{7}, \ldots$, corresponding in a natural way to the numbers $\zeta(3), \zeta(5), \zeta(7), \ldots$ Moreover, $G R T_{1}$ plays the role of the Galois group for transcendental numbers of the form $\zeta\left(k_{1}, \ldots, k_{r}\right)$, since it acts (conjecturally) on the algebra $\mathfrak{A}$ by automorphisms 49

At almost the same time, at the institute, Connes and Kontsevich had just discovered a natural occurrence of the group $G R T_{1}$ in fundamental problems of physics:

1. Connes and Kreimer 3. discovered how to make the Lie algebra $\mathfrak{g r t}_{1}$ (and other similar Lie algebras) act on the algebra corresponding to Feynman diagrams. It represents a new type of symmetry, not acting on any particular model of field theory, but sweeping away a whole class of possible Lagrangians.

2. Kontsevich 9 has recently solved the problem of quantization by deformation for Poisson manifolds. The set of possible quantizations has a symmetry group, and Kontsevich conjectures that it is isomorphic to GRT.

In both problems the numbers $\zeta\left(k_{1}, \ldots, k_{r}\right)$ arise as the values of certain integrals.

Could one dream of a better marriage, for the 40th anniversary of the Institut des Hautes Études Scientifiques and the 30th anniversary of the break with Grothendieck?

\section{Postscript (December 2000)}

The present paper was written (in French) in September 1998. I took the opportunity of the present translation into English to correct a few inaccuracies in the biographical sketch of Alexander Grothendieck. I thank the friends and colleagues who helped me to remove these inaccuracies, but I'm afraid that not everything has been corrected.

I would not alter today my views about the space problem, as expounded above. But the last section, "I have a dream", ought to be very much elaborated. During the past two years, enormous progress has been made, both in the mathematics and in the physical understanding.

From the mathematical side, we have learned to distinguish between the numerical polyzeta numbers $\zeta\left(k_{1}, \ldots, k_{r}\right)$ and their symbolic counterparts $Z\left(k_{1}, \ldots, k_{r}\right)$. These are symbols subjected only to the known polynomial relations among the $\zeta\left(k_{1}, \ldots, k_{r}\right)$. From the definitions of these numbers by series and by integrals, one derives two families of quadratic relations. Moreover, by studying carefully

\footnotetext{
${ }^{49}$ For the benefit of specialists, we mention that the preceding is a quick, informal exposition of the theory of "mixed Tate motives".
} 
the regularization of the divergent series $\zeta\left(1, k_{2}, \ldots, k_{r}\right)$, one derives a third set of relations. These three kinds of relations are the ones used for the definition of the symbolic $Z\left(k_{1}, \ldots, k_{r}\right)$. It has been announced by Jean Ecalle (Orsay, France) that the symbolic polyzetas make a free polynomial algebra $\mathfrak{A}^{\text {symb }}$. A complete proof has been given in the dissertation of my student Georges Racinet, together with the definition of a new group scheme $D M_{0}$, conjecturally identical with the group $G R T_{1}$. Much remains to be proved, for instance the transcendance conjecture, that numerical evaluation $Z\left(k_{1}, \ldots, k_{r}\right) \mapsto \zeta\left(k_{1}, \ldots, k_{r}\right)$ is an injective map from $\mathfrak{A}^{\text {symb }}$ into $\mathbb{R}$. Let us mention only that T. Rivoal $[\underline{\mathrm{R}}]$ has enormously improved on Apéry's classical result that $\zeta(3)$ is irrational (1979). I refer the reader to a forthcoming review article $[\mathrm{PC}$ on this subject.

From the physics point of view, the work of Connes and Kreimer about renormalization in quantum field theory has been deepened. The so-called "forest formula" of Zimmermann was translated into the language of Hopf algebras by Kreimer, and Connes introduced the efficient tool of Birkhoff factorization. Inspired by the theory of algebraic groups, I reformulated everything in terms of exponential of triangular matrices. Together with my student Marcus Berg, we made it a practical tool for calculation of counter-terms in renormalized field theories. At a deeper level, I was more prophetic than I had thought, and there are many reasons to believe in a "cosmic Galois group" acting on the fundamental constants of the physical theories. This group should be closely related to the group $G R T_{1}$ and might eventually help understanding of the numerical value of the fine structure constant

$$
\alpha=\frac{e^{2}}{4 \pi \varepsilon_{0} \hbar c} \sim \frac{1}{137}
$$

a long-standing puzzle.

To conclude in an optimistic mood, the "dream" I perceived two years ago as a ghost dimly visible in the fog might become true and has already motivated a lot of interesting research.

\section{REFERENCES}

[1] A. Borel, "Sur la cohomologie des espaces fibrés principaux et des espaces homogènes de groupes de Lie compacts," Ann. Math. (2) 57, 115-207 (1953). MR 14:490e

[2] H. Cartan and C. Chevalley, Géométrie algébrique, École Normale Supérieure 1955/6, Benjamin reprint.

[3] A. Connes and D. Kreimer, "Hopf algebras, renormalization and noncommutative geometry," Quantum Field Theory: Perspective and Prospective (C. DeWitt-Morette and J.-B. Zuber, eds.), Kluwer, Dordrecht, 1999, 59-109. CMP 2000:05; Commun. Math. Phys. 199, 203-242 (1998). MR 99h:81137

[4] H. Crapo and G. C. Rota, On the Foundations of Combinatorial Theory: Combinatorial Geometries, MIT Press, Cambridge, MA (1970). MR 45:74

[5] P. Deligne, "Catégories tannakiennes," in: Grothendieck Festschrift, Vol. II, 111-195. Progress in Math., Vol. 87, Birkhäuser, Boston (1990). MR 92d:14002

[6] S. Doplicher and J. E. Roberts, "Endomorphisms of $C^{*}$-algebras, cross-products and duality for compact groups," Ann. Math. (2) 130, 75-119 (1989). MR 90j:46058

[7] A. Grothendieck, "Produits tensoriels topologiques et espaces nucléaires," Mem. Amer. Math. Soc. No. 16 (1955). MR 17:763c Sem. Bourbaki, Vol. 2, Exp. No. 69, 193-200 (1995). CMP 98:09

[8] A. Grothendieck, "Résumé de la théorie métrique des produits tensoriels topologiques," Bol. Soc. Mat. 8, São Paulo, 1-79 (1953). MR 20:1194

[9] M. Kontsevich, "Quantization deformation of Poisson manifolds I," $q$-alg 9709040. 
[10] L. Schwartz, Un mathématicien aux prises avec le siècle, Odile Jacob, Paris (1997). CMP 98:17

[11] J.-P. Serre, "Faisceaux algébriques cohérents," Ann. Math. (2) 61, 197-278 (1955). MR 16:953c

[12] J. Tapia, "Quelques spectres en $K$-théorie topologique des algèbres de Fréchet et applications à l'algèbre des fonctions de classe $C^{\infty}$ sur une variété," preprint IHÉS/M/91/37.

[13] A. Weil, "Théorie des points proches sur les variétés différentiables," Colloque de Géométrie Différentielle (Strasbourg, 1953), C.N.R.S., 111-117. MR 15:828f =Oeuvres Scientifiques, vol. II, Springer-Verlag, 1980, 103-109.

[14] D. Zagier, "Values of zeta functions and their applications," Progress in Math., 120, Birkhäuser, Basel, 497-512 (1994). MR 96k:11110

Fundamental works:

[T] A. Grothendieck et al., Théorie des topos et cohomologie étale des schémas, Springer Lect. Notes in Math., 269, 270, 305 (1972/3). MR 50:7130: MR 50:7131] MR 50:7132

[NC] A. Connes, Noncommutative Geometry, Academic Press (1994). MR 95j:46063

\section{Supplementary Bibliography (ADDED IN PROOF)}

[PC] P. Cartier, "Fonctions polylogarithmes, nombres polyzétas et groupes unipotents" (to appear in the Bourbaki Seminar, year 2000-1).

[R] T. Rivoal, "La fonction zêta de Riemann prend une infinité de valeurs irrationnelles aux entiers impairs", C.R. Acad. Sci. Paris, series I, 267-270 (2000). CMP 2001:02

ENS-DMA, 45 Rue d'Ulm, 75230 Paris Cedex 05, France

E-mail address: cartier@ihes.fr 